Original Research

\title{
Study of the Relationship Between Ergonomic Risk Level and Job Stress in the Employees of a Food Production Plant
}

\author{
Fateme Chaharaghran $^{1}$, Shahnaz Tabatabaei ${ }^{* 2}$
}

1. MSc in Ergonomics, Department of Ergonomics, School of Public Health and Safety, Shahid Beheshti University of Medical Sciences, Tehran, Iran

2. Associate Professor, Department of Ergonomics, School of Public Health and Safety, Shahid Beheshti University of Medical Sciences, Tehran, Iran

\begin{tabular}{ll}
\hline \multicolumn{2}{c}{ Article Info } \\
\hline Original Article \\
Received: & $2018 / 10 / 01$ \\
Accepted: & $2019 / 02 / 01$ \\
Published Online: & $2019 / 03 / 21$ \\
DOI: $10.30699 /$ jergon.7.1.28
\end{tabular}

Use your device to scan and read the article online

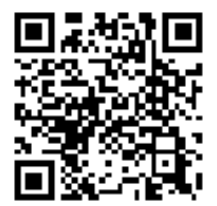

Corresponding Information

Shahnaz Tabatabaei,

Associate Professor, Department of Ergonomics, School of Public Health and Safety, Shahid Beheshti University of Medical Sciences, Tehran, Iran

\begin{abstract}
Background and Objectives: Considering that musculoskeletal disorders and occupational stress are one of the most important reasons for disability, physical and mental problems in the staff, the study of their causes is of great importance. Therefore, this study was conducted to investigate the relationship between ergonomic risk level and job stress in employees of a food production plant.
\end{abstract}

Methods: This was a descriptive-analytic research. It was carried out for a sample of 200 employees in the production and packaging of a food production plant in 2018. The QEC method was used to determine the ergonomic risk level and the OSPU questionnaire was used to measure occupational stress. Descriptive and inferential statistics ( $\mathrm{T}$ and $\mathrm{F}$ tests) were used to analyze the data.

Results: The findings showed that the level of ergonomic risk in the neck, shoulders and wrists is in the unfavorable situation. Workstations will need to be repaired in the near future. There is a significant relationship between the level of ergonomic risk and the demographic variables of gender and education with job stress.

Conclusion: Unfavorable ergonomic status of workstations in the food factory has increased the worker's stress in the manufacturing and packaging sectors. Therefore, proper design of workstations and consideration of ergonomic principles are effective in improving the physical and mental health of employees.

Keywords: Work posture; Job stress; Food industry

\section{Email:}

tabshahnaz@yahoo.com

Copyright (C) 2019, This is an original open-access article distributed under the terms of the Creative Commons Attribution-noncommercial 4.0 International License which permits copy and redistribute of the material just in noncommercial usages with proper citation.

How to Cite This Article:

Chahraghran F, Tabatabaei S. Study of the Relationship Between Ergonomic Risk Level and Job Stress in the Employees of a Food Production Plant. Iran J Ergon. 2019; 7 (1) :28-36 


\title{
E-ISSN: 2345-5365 | مجلة اركونومى | سال \، شمارهٔ ا، بهار
}

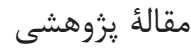 \\ بررسى ارتباط بين سطح ريسك ارگونوميكى و تنيدكى شغلى در كاركنان يك كارخانه \\ توليد مواد غذايى تونى تئي \\ فاطمه جههاراقران'، شهناز طباطبايى r"
}

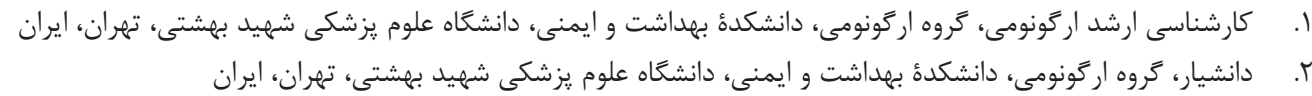

\begin{tabular}{|c|}
\hline جكيد \\
\hline 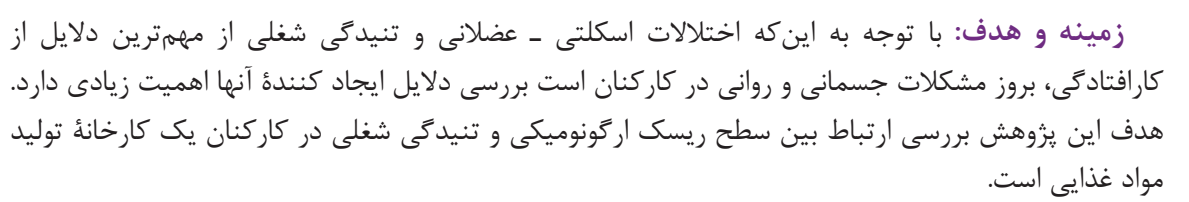 \\
\hline 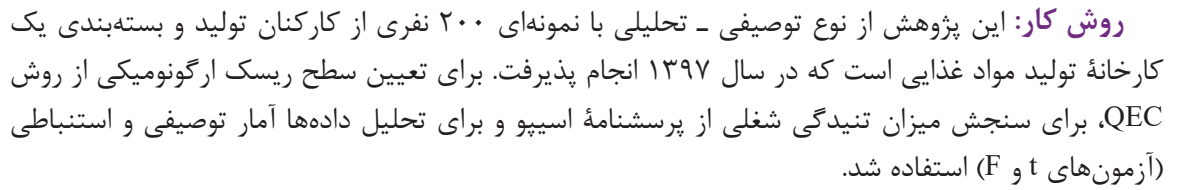 \\
\hline 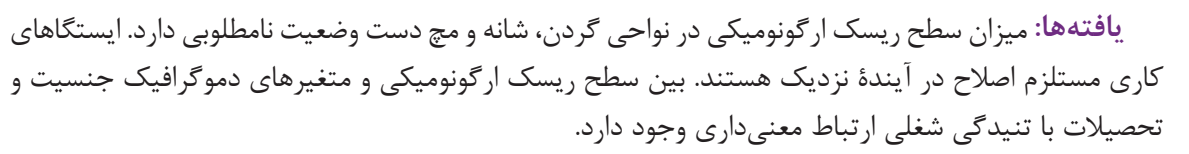 \\
\hline 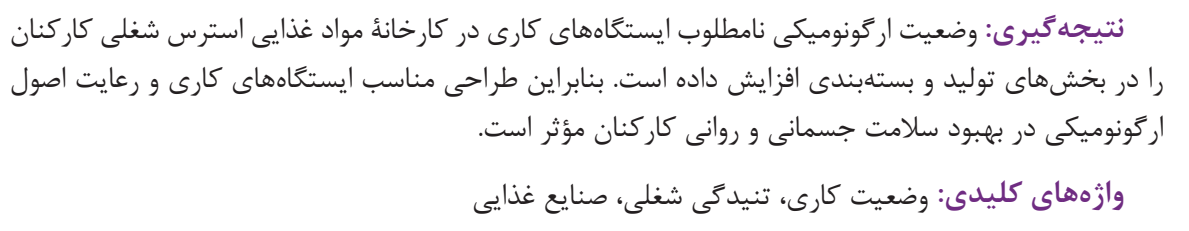 \\
\hline
\end{tabular}

اطلاعات مقاله

تاريخ وصول: : ت

تاريخ يذيرش:

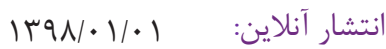

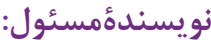

شهناز طباطبايى

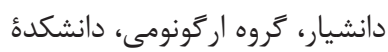

بهداشت و ايمنى، دانشگاه علوم

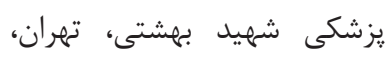

ايران

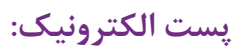

tabshahnaz@yahoo.com

تماس با يك ماده خطرناك مشخص است، اختلالات اسكلتى

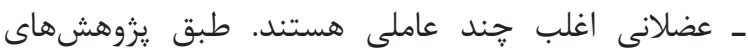

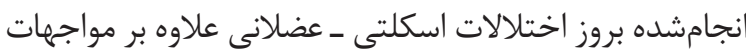

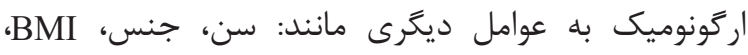

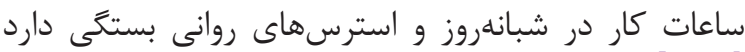

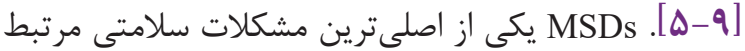

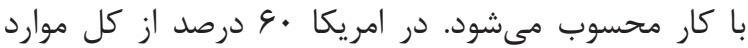

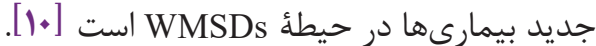
اغلب يزوهشگران معتقدند كه عوامل فيزيكى فقط

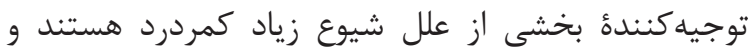

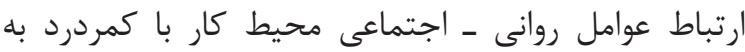

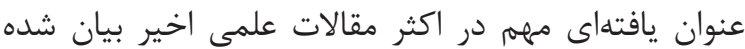

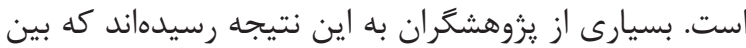

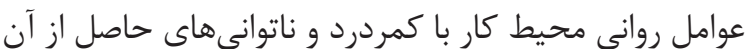

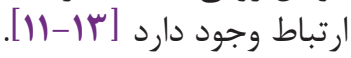
از مشخصات سازمان سالم آن است كه سلامت جسمانى و

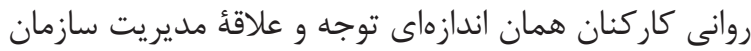

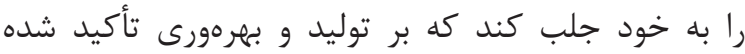

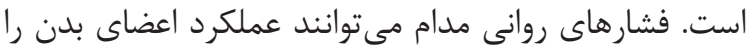

كاركنان مشاغل مختلف بهخصوص صنايع غذايى از وضعيت كارى نامناسب رنج مىبرند و مشكلات جسمان مخانى

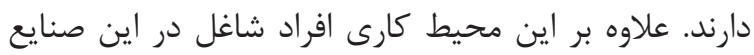

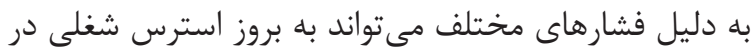

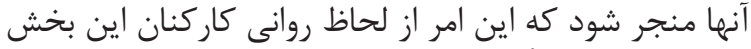
صنعتى را تحت تأثير قرار مىدهد أند إند اختلالات اسكلتى - عضلانى ناشى از مار (Work-related Musculoskeletal Disorders) (WMSDs) علائمى كَفته مىشود كه توسط ريسك فاكتورهاى شغلى

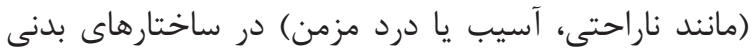

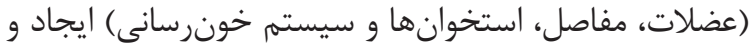

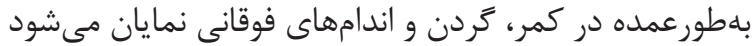

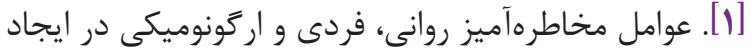

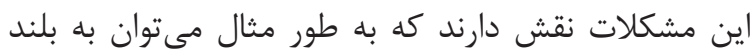

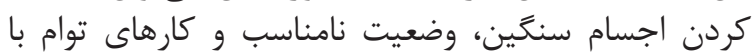
حركات تكرارى اشاره كرد [r-r.r. برخلاف بسيارى از بيمارىهاى ناشى از كار كه منشأ آنها 
Keyserling و همكاران (1919) با هدف ارزيابى استرس

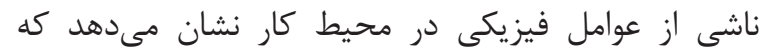

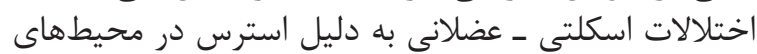

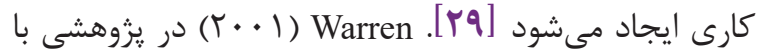

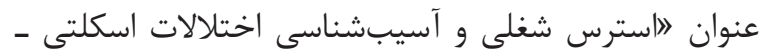

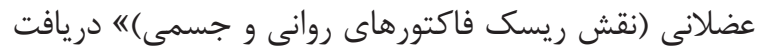

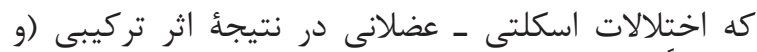

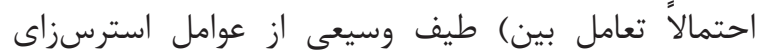

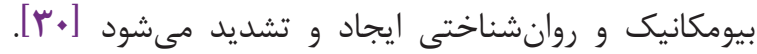

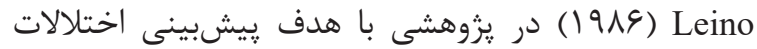

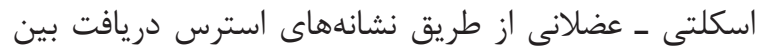

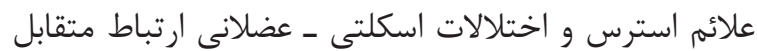

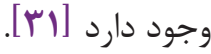

برخلاف گسترش روزافزون فريندهاى خودكار هنوز بخش

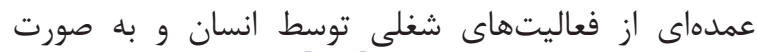

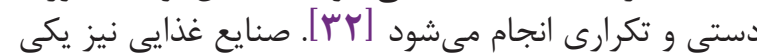

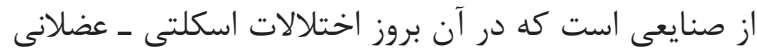

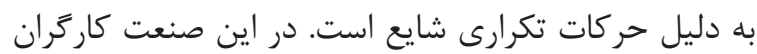

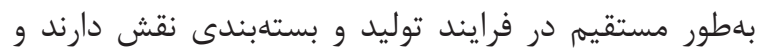

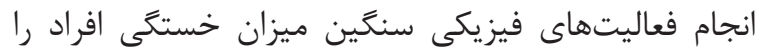

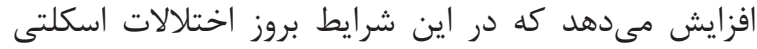

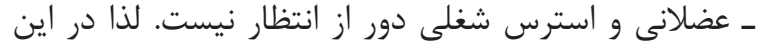

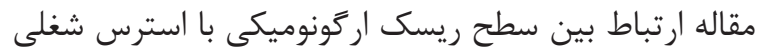

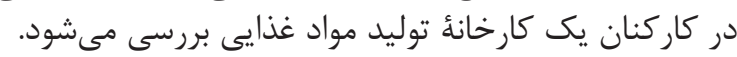

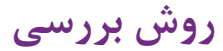

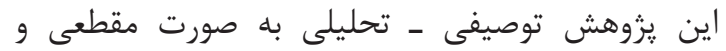

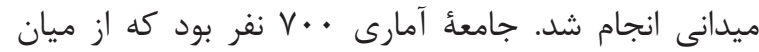

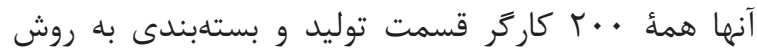

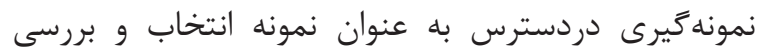

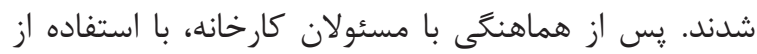

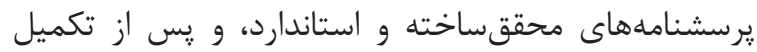

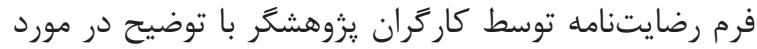

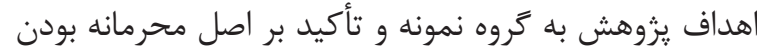

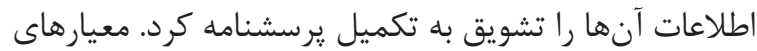

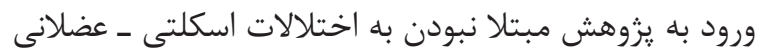

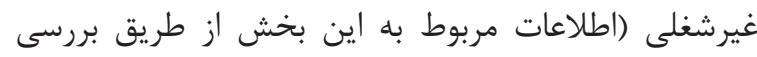

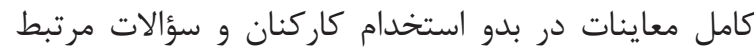

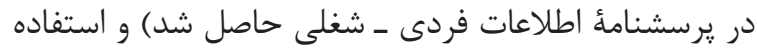

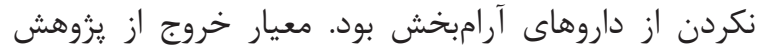

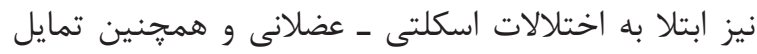

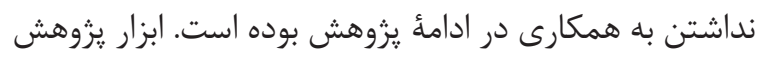

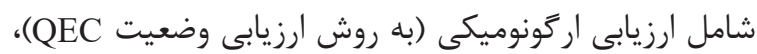

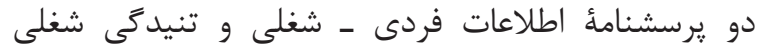
(استرس شغلى) اسييو بود كه به اين شرح است:

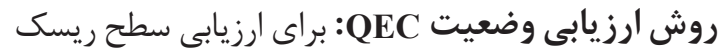

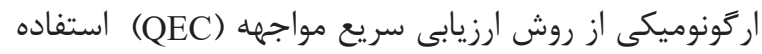

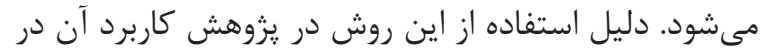

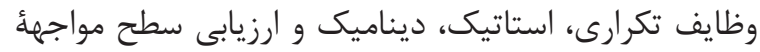

تغيير دهند. در صورت تكرار زياد تغييرات ساختارى در بدن

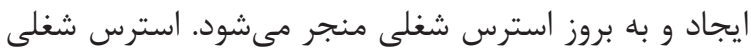

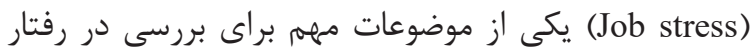

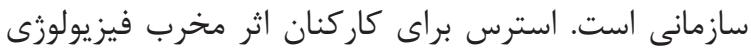

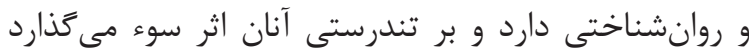

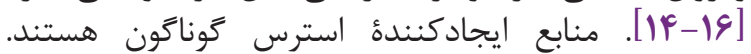

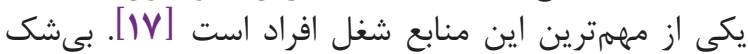

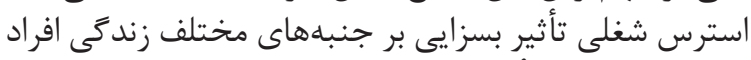

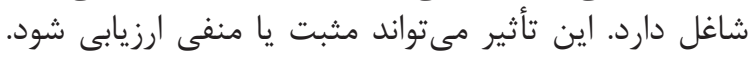

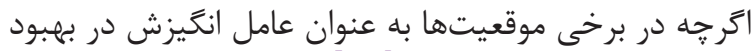

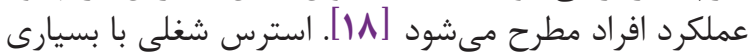

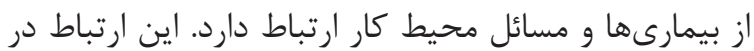

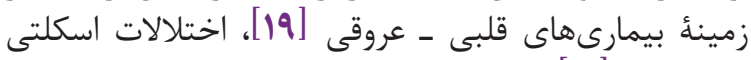

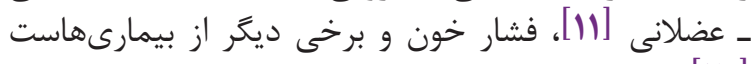

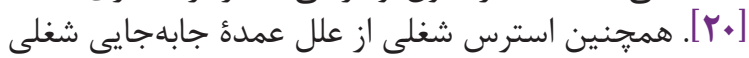

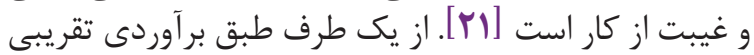

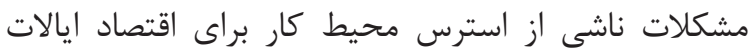

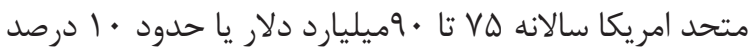

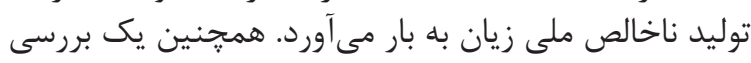

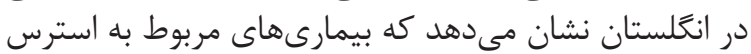

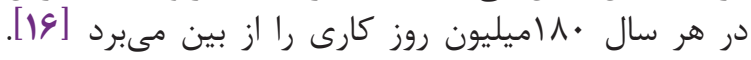

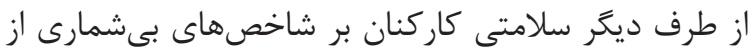

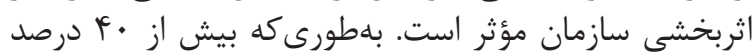

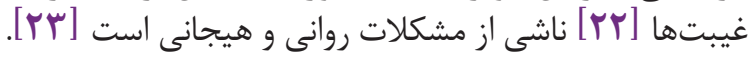
يolghanabadi

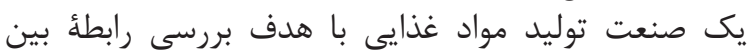

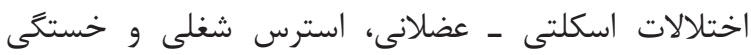

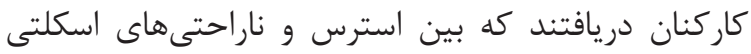

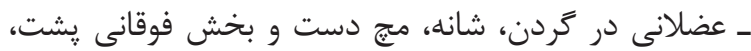

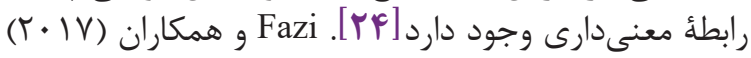

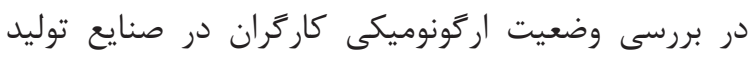

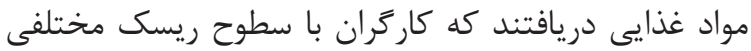

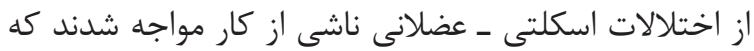

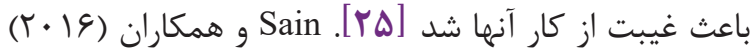

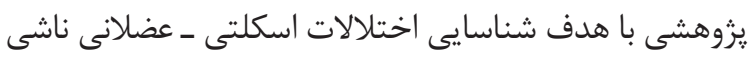

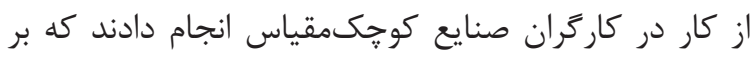

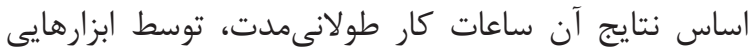

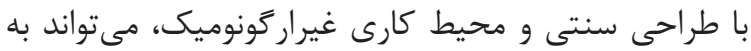

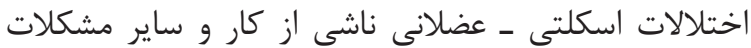

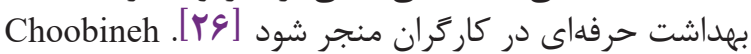

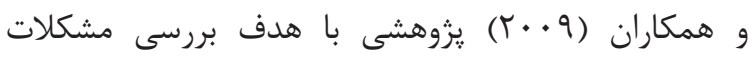

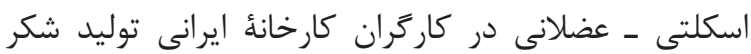

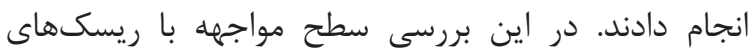

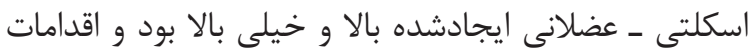

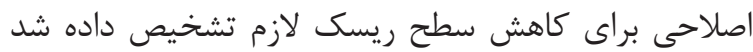

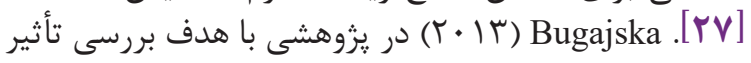

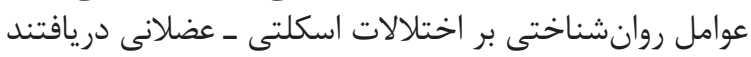

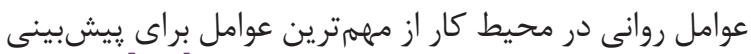

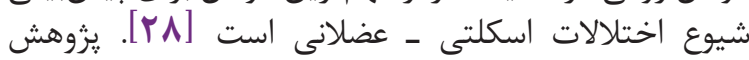


نمرهذارى : يرسشنامة استرس شغلى اسيبو بر مبناى

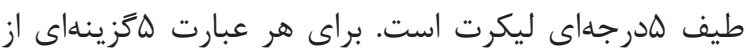

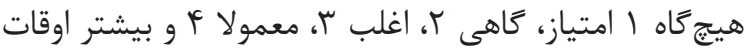

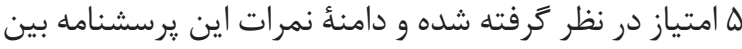

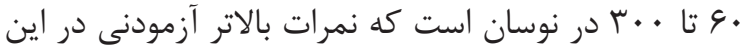

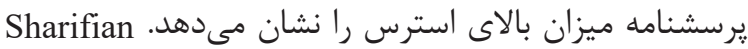

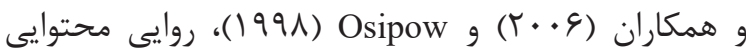

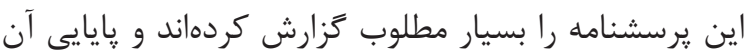

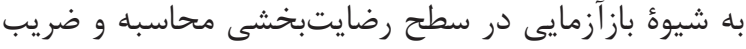

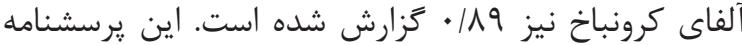

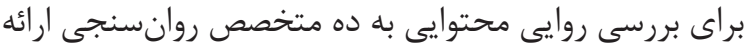

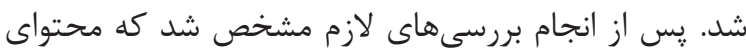

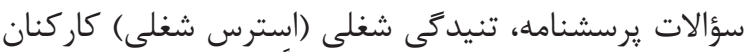

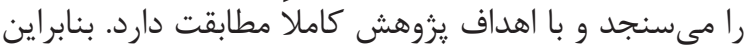

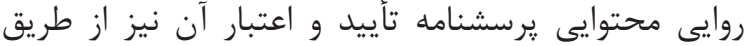

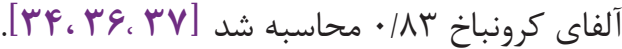

تجزيهوتحليل دادها: دادهها با استفاده از آمار توصيفى

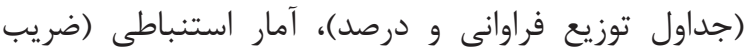

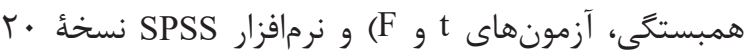
تجزيهوتحليل شدند. (SPSS Inc., Chicago, Ill., USA)

\section{يافتهها}

يافتهاى توصيفى مربوط به متغيرهاى جمعيتشناختى

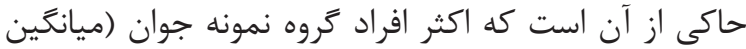

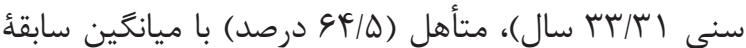

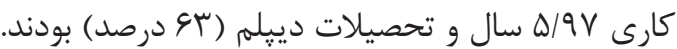

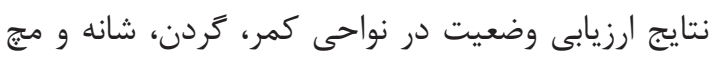

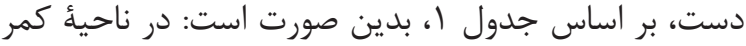

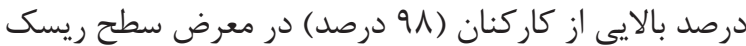

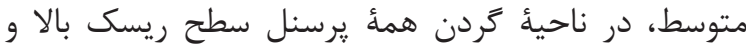

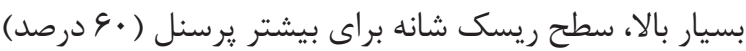

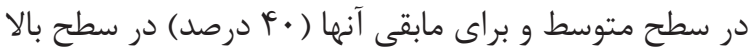

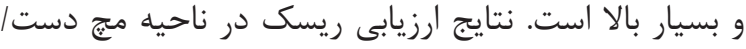

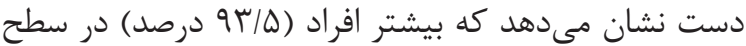

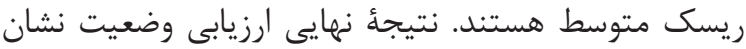

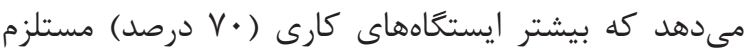
اصلاح در آيندهُ نزديك هستند

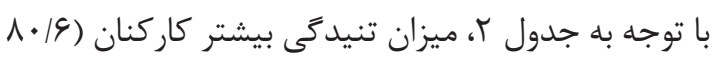
درصد) در سطح متوسط تا شديد و شديد است.

بر اساس جدول س، بين وضعيت كارى و تنيدگى شغلى

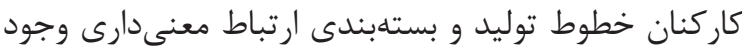

با توجه به جداول f أو ه، بين تنيد

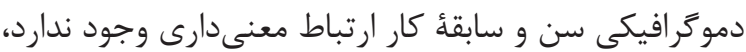

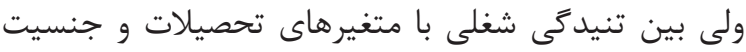

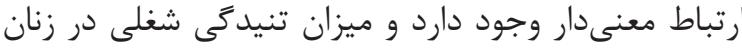
بهطور معنى اردارى بيشتر از مردان است.
كل بدن است. لذا با توجه به شرايط كارى كاركنان كه مستلزم

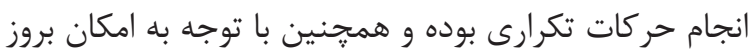

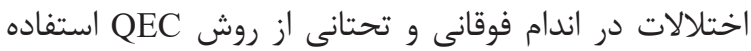

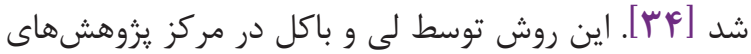

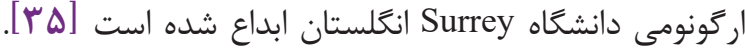

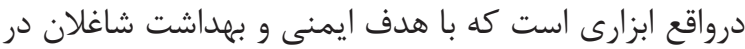

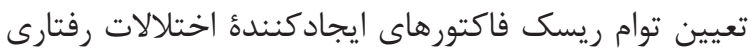

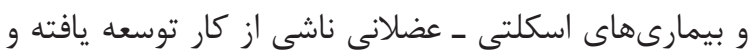

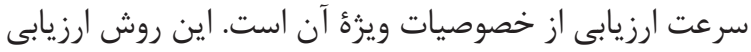

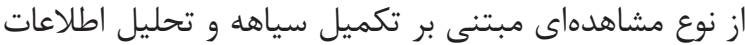

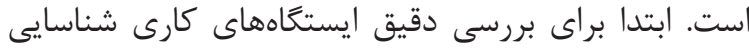

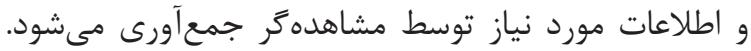
سيس وظايف مختلف كارى بازديد، شناسايى و ثبت شئ شده

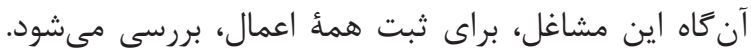

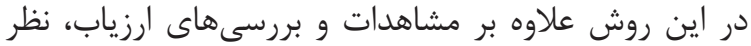

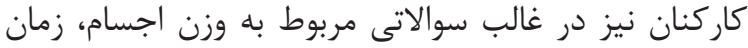

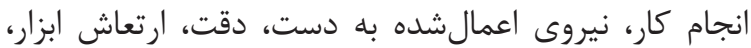

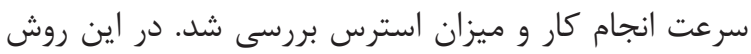

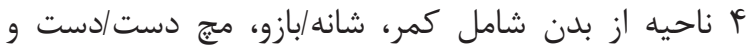

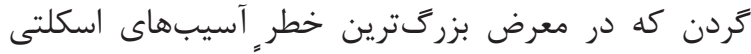

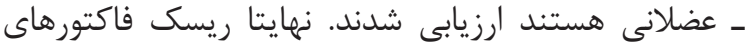

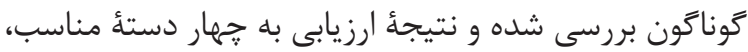

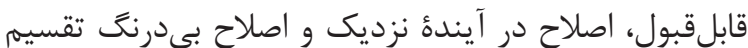

شد [سr]].

يرسشنامهُ اطلاعات فردى ـ شغلى: اين : شرسشنامه

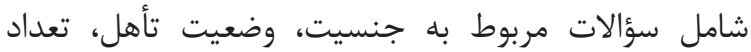

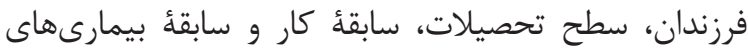
اسكلتى - عضلانى بود.

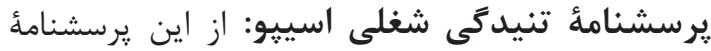

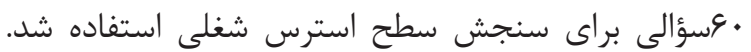

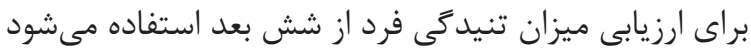
كه عبارت است ازئ مئن

1. باركارى نقش: بررسى وضعيت شخص نسبت به تقاضاهاى محيط كار؛

r. بى كفايتى نقش: ارزيابى تناسب ميزان مهارت،

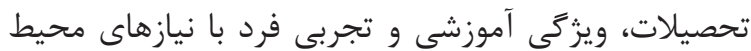
كار؛ تص

ז. دوگانتىى نقش: بررسى آَاهى فرد از اولويتها، جشمداشتهاى محيط كار و معيارهاى ارزشيابى؛ نقي؛ أ. محدوده نقش: ارزيابى تضادهايى كه از وجدان كارى نقى

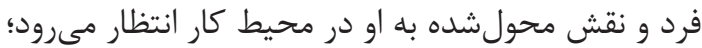
ه. مسئوليت: سنجش احساس مسئوليت فرد از نظر كارايى و رفاه ديخران در محيط كار؛ سئ

9. محيط فيزيكى: بررسى شرايط نامساعد فيزيكى محيط كارى كه فرد در معرض آن قرار دارد. 
جدول ا. توزيع فراوانى كاركنان بر حسب امتياز ارزيابى سطح ريسك با استفاده از روش QEC

\begin{tabular}{|c|c|c|c|}
\hline درصد فراوانى & فراوانى & سطح ريسك & ناحيه \\
\hline r & f & هايين & \multirow{2}{*}{ 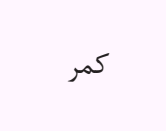 } \\
\hline 91 & 199 & متوسط & \\
\hline ६. & IT. & متوسط & \multirow{3}{*}{ شانه/بازو } \\
\hline rq/ब & vq & بالا & \\
\hline$\cdot 10$ & 1 & بسيار بالا & \\
\hline $9 \pi / \Delta$ & IAV & متوسط & \multirow{2}{*}{ مج دست/دست } \\
\hline $8 / 0$ & זו & بالا & \\
\hline$\Delta \Delta / \Delta$ & 111 & بالا & \multirow{2}{*}{ كردن } \\
\hline$F F / D$ & 19 & بسيار بالا & \\
\hline$r \Delta / \Lambda$ & $\Delta V$ & انجام يزوهشهاى بيشتر & \multirow{3}{*}{ امتياز نهايى } \\
\hline$\checkmark \cdot$ & If. & اصلاح در آينده & \\
\hline $1 / 0$ & r & اصلاح بىدرنگ & \\
\hline
\end{tabular}

$$
\text { جدول r. توزيع فراوانى كاركنان بر حسب تنيدكى شغلى }
$$

تنيدكى شغلى

$$
\text { جدول r. ارتباط بين سطح ريسك ارَونوميكى با تنيدگى شغلى از طريق آزمون دقيق فيشر }
$$

\section{تنيدَّى شغلى تعداد (درصد)}

\section{P-value}

شديد

متوسط تا شديد

كم تا متوسط

سطح ريسك

متغير

$(\cdot) \cdot$

$(Y / \Lambda))^{\prime}$

$(\cdot) \cdot$

קايين

.199

$(1 \cdot \cdot) 11$

$(9 V / T r) I T V$

$(1 \cdots)$ Fl

متوسط

$(1 \cdot \cdot) 11$

$(1 \cdots)|4|$

(1..) \&

\begin{tabular}{|c|c|c|}
\hline \multirow{3}{*}{$\cdot / 4 q$} & $(\Delta \mathcal{F} / \mathcal{F} \Lambda) \mathcal{G}$ & $(\Delta \wedge / T r) \wedge T$ \\
\hline & $(q \Delta / F q) \Delta$ & $(f \mid / \vee \wedge) \Delta q$ \\
\hline & $(1 \cdots) 11$ & $(1 \cdots)|f|$ \\
\hline
\end{tabular}

كل

$\left(9 N / T^{\prime}\right)$ TN

متوسط

rTI/VT)

بالا و بسيار بالا

شانه

$(1 \cdot \cdot) 11$

$(1 \cdots)|f|$

(1..) Fl

كل 
تنيدَّى شغلى تعداد (درصد)

\begin{tabular}{|c|c|c|c|c|c|}
\hline P-value & شديد & متوسط تا شديد & كم تا متوسط & سطح ريسك & متغير \\
\hline \multirow{3}{*}{. Mt } & $(9 \cdot / 94) 1$. & $(9 T / T I) \mid r$. & $(q \vee / q \mu) \mathcal{F}$. & متوسط & \multirow{3}{*}{ مج دست } \\
\hline & $(9 / 1 \Gamma) 1$ & $(\mathrm{~V} / \mathrm{VG}) \backslash 11$ & $\left(T / T^{\mathcal{L}}\right) 1$ & بالا & \\
\hline & $(1 \cdots) 11$ & $(1 \cdots)|f|$ & $(1 \cdots){ }_{1}$ & كل & \\
\hline \multirow{3}{*}{$<\cdot|\cdot \cdot|$} & $(\& \Gamma / \Delta q) \vee$ & $(\varphi \& / V \wedge) \& \varphi$ & r & بالا & \multirow{3}{*}{ كردن } \\
\hline & $(r \varepsilon / 4) f^{q}$ & $(\Delta T / Y T) V \Delta$ & $(19 / 01) \wedge$ & بسيار بالا & \\
\hline & $(1 \cdots) 11$ & $(1 \cdots)|f|$ & $\left.(1 \cdots)\right|_{1}$ & كل & \\
\hline \multirow{4}{*}{.$/ \cdot 1$} & $(\Gamma \varepsilon / \mu q) \uparrow$ & (YT) MI & $(\lceil\wedge / \Lambda I) T$. & يزوهش هاى بيشتر & \multirow{4}{*}{ امتياز نهايى } \\
\hline & $(\Phi \mu / \Delta \Lambda) V$ & $(V \Delta / q Y)) \cdot V$ & $(\Delta I / T r) Y I$ & اصلاح درآينده & \\
\hline & $(\cdot) \cdot$ & $(T / I T) r$ & $(\cdot) \cdot$ & اصلاح بىدرنت & \\
\hline & $(1 \cdots) 11$ & $(1 \cdot \cdot)|f|$ & $(1 \cdots) f_{1}$ & كل & \\
\hline
\end{tabular}

جدول F. ارتباط تنيدكى شغلى و متغير هاى دموكر افيكى سن و سابقُ كار

\begin{tabular}{|c|c|c|c|c|}
\hline P-value & آمارةٌ آزمون & مقدار OR نسبت به ردهُ هايه & متغير هاى كمكى & تنيدكى شغلى \\
\hline$\cdot 1 \cdot \Delta$ & r/AF & $\cdot 190$ & سن & \multirow{2}{*}{ متوسط تا شديد } \\
\hline$\cdot \pi \Delta$ & $\cdot / \Lambda \Delta$ & $1 / \cdot \Delta$ & سابقهُ كار & \\
\hline$\cdot \mid \Delta \Lambda$ & $\cdot|r|$ & $\cdot / 97$ & سن & \multirow[b]{2}{*}{ } \\
\hline$\cdot / r$ & 1/94 & $1 / 11$ & سابقهُ كار & \\
\hline
\end{tabular}

جدول ه. ارتباط بين تنيدكى شغلى با متغيرهاى دموكرافيكى جنسيت و تحصيلات از طريق آزمونهاى كاى اسكور و دقيق فيشر

\section{تنيدكَ شغلى تعداد (درصد)}

\begin{tabular}{|c|c|c|c|c|c|}
\hline P-value & شديد & متوسط تا شديد & كم تا متوسط & سطح ريسك & \\
\hline \multirow{3}{*}{$\cdot 1 \cdot r$} & $(\xi \Psi / \xi Y) \vee$ & $(V T / K T) 1 \cdot r$ & $(\mathcal{G T / q T ) \backslash \Lambda}$ & زن & \multirow{3}{*}{ جنسيت } \\
\hline & $(r g / r \Lambda) F$ & $(T V / V T) Y q$ & זr (D\&/II & مرد & \\
\hline & $(1 \cdots) 11$ & $(\mid \cdots)|f|$ & $\left.(1 \cdots)\right|_{1}$ & كل & \\
\hline \multirow{4}{*}{$\cdot / \cdot r$} & $(F \Delta / \Delta \cdot) \Delta$ & $(r \cdot)$ TA & $(q \backslash / F q) \mid V$ & سيكل & \multirow{4}{*}{ تحصيلات } \\
\hline & $(f \Delta / \Delta \mid) \Delta$ & $(\varphi q / r \wedge) q \vee$ & $(\{\wedge / \Lambda I) \zeta$. & دييلهم & \\
\hline & $(9 / 1 T) 1$ & $(1 \cdot / V T) \mid \Delta$ & $(9 / \vee q) F$ & ليسانس & \\
\hline & $(1 \cdots) 11$ & $(\mid \cdots)|f|$ & $\left.(1 \cdots)\right|_{1}$ & كل & \\
\hline
\end{tabular}


معنى دارى بيشتر از مردان است. يزوهش Givaryan و

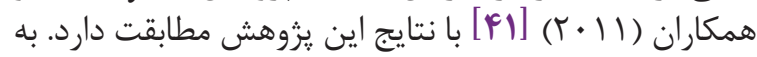

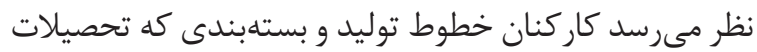

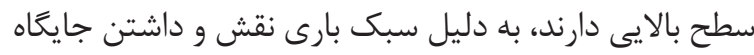

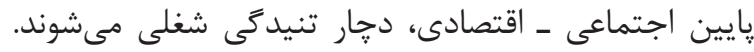

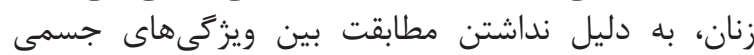

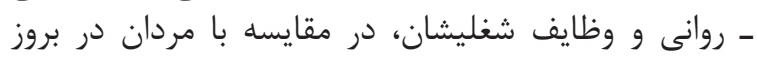

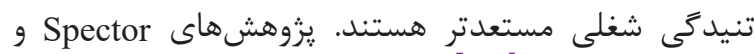

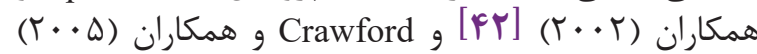

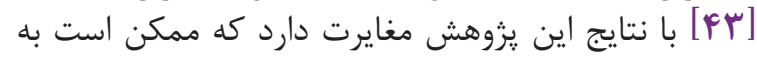
دليل تفاوت در وضعيت اقتصادى و شرايط محيط كار خروهنها باشد.

\section{نتيجه}

طراحى نامناسب ايستكاههاى كارى و وجود وضعيتهاى

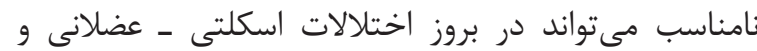

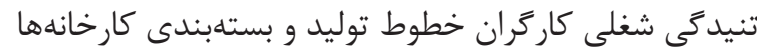

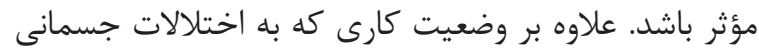

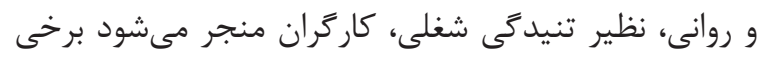

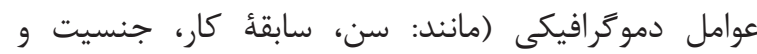

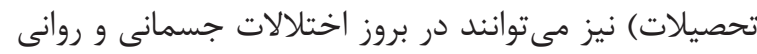

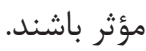

بنابراين طراحى اركونوميكى ايستخاههاى كارى و واصلاح

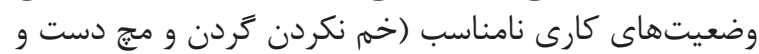

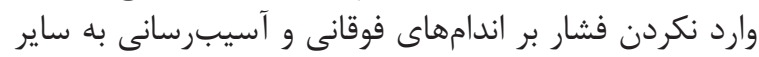

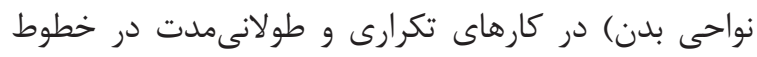

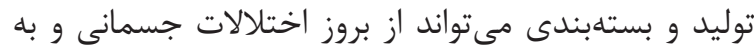

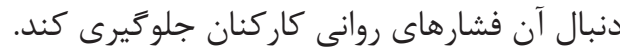

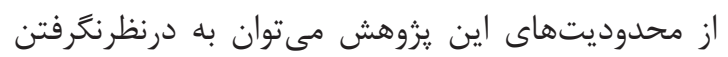

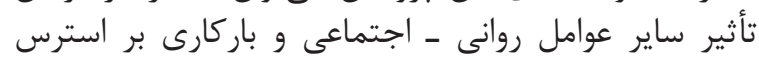

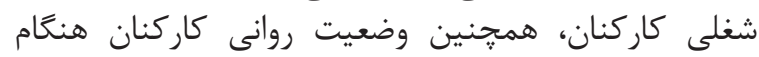

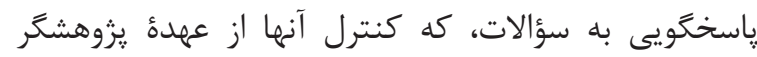
خارج بود، اشاره كرد. - الة

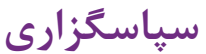

بدين وسيله مراتب تشكر و سياس خود را از مدرئ مديريت

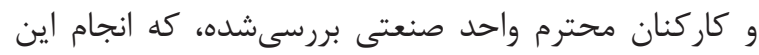
يزؤهش بدون همكارى ايشان ميسر نبود، اعلام مى داريمه كه انجام اين.

$$
\text { تعارض در منافع }
$$

بين نويسندًان هيجَّونه تعارضى در منافع وجود ندارد.

\section{بحث}

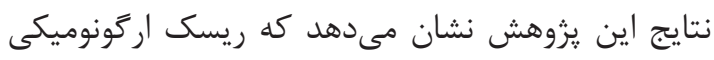

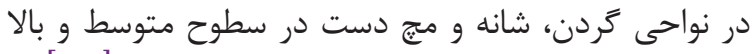

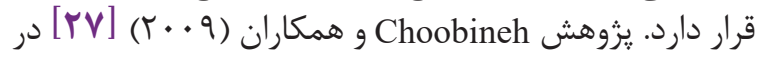

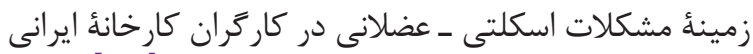

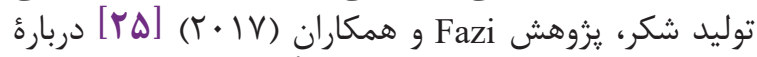

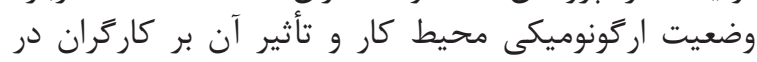

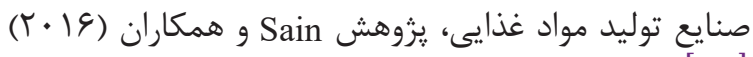

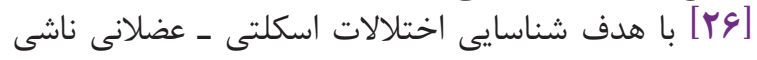

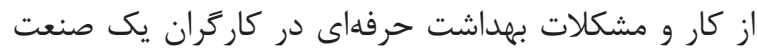

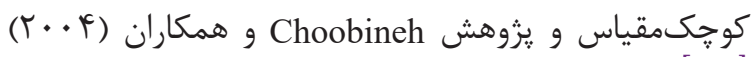

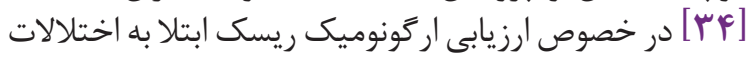

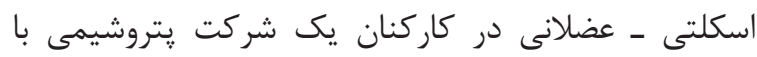

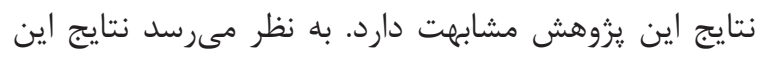

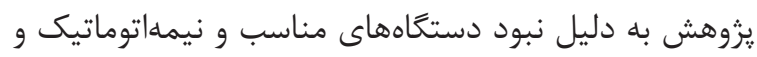

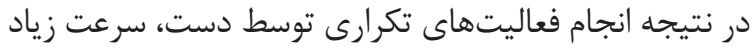

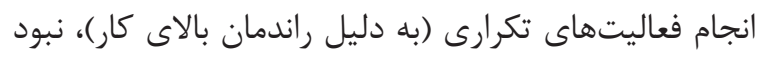

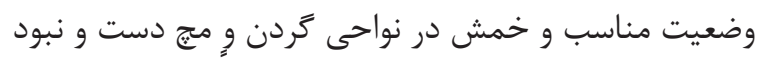

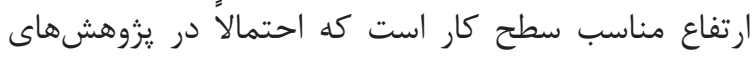

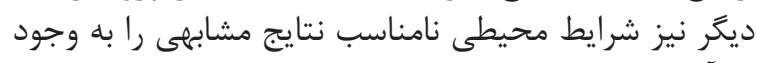

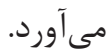

بين امتياز نهايى وضعيت كارى و تنيدگى شغلى شعلى كاركنان

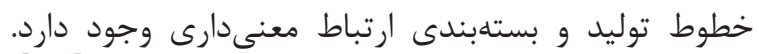

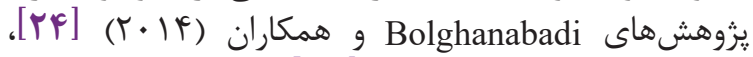
و Bugajska

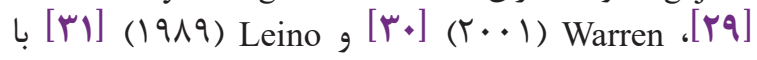

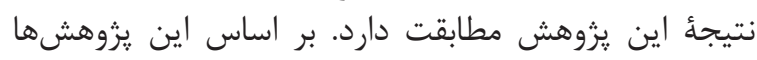

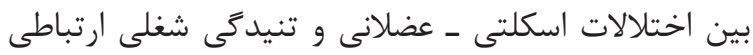

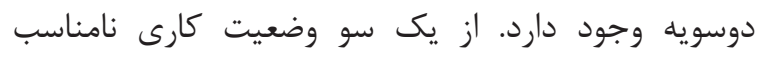

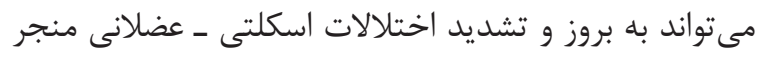

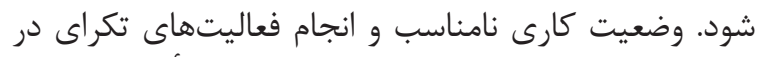

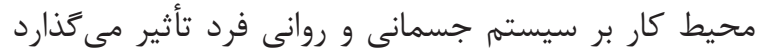

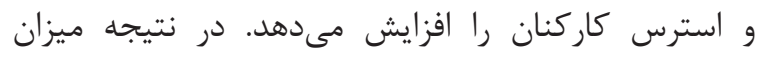

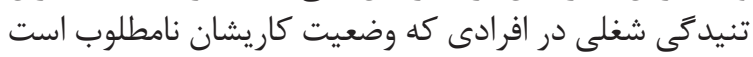

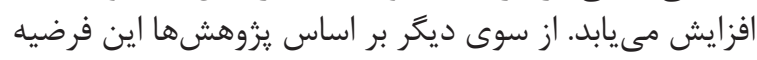

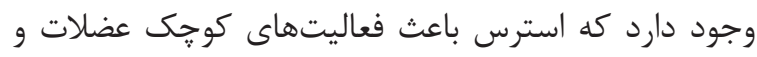

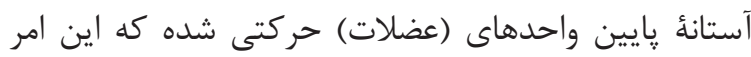

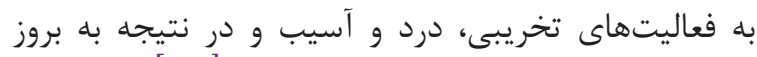

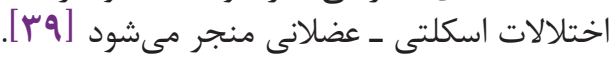

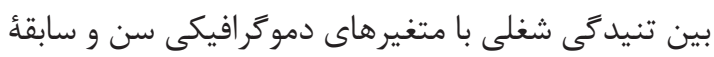

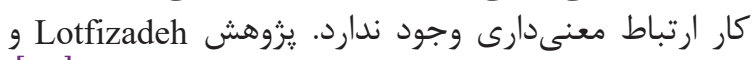

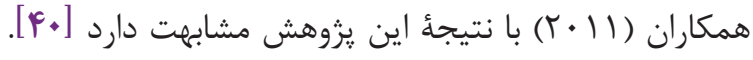

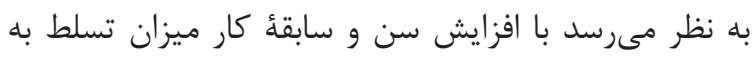

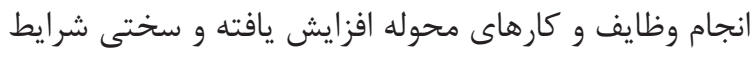
محيط كار و در نتيجه فشار روانى كاهش مي ميابد.

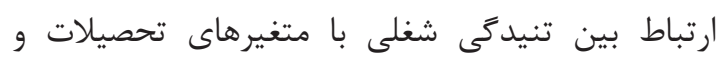

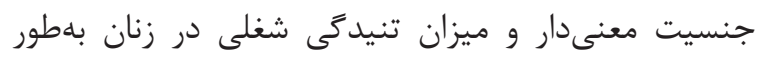




\section{References}

1. Kuorinka I, Jonsson B, Kilbom A, Vinterberg H, Biering-Sørensen F, Andersson G, Jørgensen K. Standardised Nordic questionnaires for the analysis of musculoskeletal symptoms. Applied ergonomics. 1987 Sep 1;18(3):233-7.

2. Bernard BP, Putz-Anderson V. Musculoskeletal disorders and workplace factors; a critical review of epidemiologic evidence for work-related musculoskeletal disorders of the neck, upper extremity, and low back. 1997.

3. Krause N, Dasinger LK, Deegan LJ, Rudolph L, Brand RJ. Psychosocial job factors and return-towork after compensated low back injury: A disability phase-specific analysis. American journal of industrial medicine. 2001 Oct;40(4):374-92.

4. da Costa BR, Vieira ER. Risk factors for work-related musculoskeletal disorders: a systematic review of recent longitudinal studies. American journal of industrial medicine. 2010 Mar;53(3):285-323.

5. Cabeças JM. Occupational musculoskeletal disorders in Europe: impact, risk factors and preventive regulations. 2006.

6. Peele PB, Xu Y, Colombi A. Medical care and lost work day costs in musculoskeletal disorders: older versus younger workers. InInternational Congress Series 2005 Jun 1 (Vol. 1280, pp. 214218). Elsevier.

7. Koo HR, Shin YS, Chae HS, Lee KS. The research of job stress and MSDs symptoms of small plants with agricultural products. Journal of Agricultural Extension \& Community Development. 2011;18(4):861-77.

8. Choobineh A, Nouri E, Arjmandzadeh A, Mohamadbaigi A. Musculoskeletal disorders among bank computer operators. Iran occupational health. 2006 Oct 15;3(2):3-0.

9. Hootman JM, Sniezek JE, Helmick CG. Observations from the CDC: Women and Arthritis: Burden, Impact, and Prevention Programs. Journal of women's health \& gender-based medicine. 2002 Jun 1;11(5):407-16.

10. Vanwonterghem K. Work-related musculoskeletal problems: Some ergonomic considerations. Journal of human ergology. 1996 Jun;25(1):5-13.

11. Yip YB. A study of work stress, patient handling activities and the risk of low back pain among nurses in Hong Kong. Journal of advanced nursing. 2001 Dec;36(6):794-804.

12. Yip VY. New low back pain in nurses: work activities, work stress and sedentary lifestyle. Journal of advanced nursing. 2004 May;46(4):430-40.
13. Feyer AM, Herbison P, Williamson AM, de Silva I, Mandryk J, Hendrie L, Hely MC. The role of physical and psychological factors in occupational low back pain: a prospective cohort study. Occupational and environmental medicine. 2000 Feb 1;57(2):116-20.

14. Munro L, Rodwell J, Harding L. Assessing occupational stress in psychiatric nurses using the full job strain model: the value of social support to nurses. International Journal of Nursing Studies. 1998 Dec 1;35(6):339-45.

15. Zimber A. Workload and stress in caring for the elderly: status of research and research agenda. Zeitschrift fur Gerontologie und Geriatrie. 1998 Dec;31(6):417-25.

16. Muscroft J, Hicks C. A comparison of psychiatric nurses' and general nurses' reported stress and counselling needs: a case study approach. Journal of advanced nursing. 1998 Jun;27(6):1317-25.

17. Mcgrath A, Reid N, Boore. Occupational stess nursing.Int J Nurse Stud. 2003; 40(5):555-65.

18. Breslow MJ. The role of stress hormones in perioperative myocardial ischemia. International anesthesiology clinics. 1992;30(1):81-100.

19. Thorbjörnsson CO, Alfredsson L, Fredriksson K, Köster M, Michélsen H, Vingård E, Torgen M, Kilbom A. Psychosocial and physical risk factors associated with low back pain: a 24 year follow up among women and men in a broad range of occupations. Occupational and environmental medicine. 1998 Feb 1;55(2):84-90.

20. Winkleby MA, Ragland DR, Syme SL. Self-reported stressors and hypertension: evidence of an inverse association. American Journal of Epidemiology. 1988 Jan 1;127(1):124-34.

21. Parker PA, Kulik JA. Burnout, self-and supervisor-rated job performance, and absenteeism among nurses. Journal of Behavioral Medicine. 1995 Dec 1;18(6):581-99.

22. Fielding J. A comparison of hospital and community based mental health nurses perception of their work environment and psychological state. J Adv Nurs 1994; 1198-1204.

23. Schaefer JA, Moos RH. Effects of work stressors and work climate on long-term care staff's job morale and functioning. Research in nursing \& health. 1996 Feb;19(1):63-73.

24. Bolghanabadi S, Pour M. The relationship between musculoskeletal disorders, stress and fatigue in the food industry employees. Iranian Journal of Ergonomics. 2014 Jun 15;2(1):54-63.

25. Fazi HM, Mohamed NM, Ab Rashid MF, Rose 


$$
\text { جهاراقران و طباطبايى ا بررسى ارتباط بين سطح ريسك اركونوميكى و تنيدگى شغلى.... }
$$

AN. Ergonomics study for workers at food production industry. InMATEC Web of Conferences 2017 (Vol. 90, p. 01003). EDP Sciences.

26. Sain MK, Meena ML. Occupational health and ergonomic intervention in Indian small scale industries: a review. Int J Recent Adv Mechanical Engin. 2016;5(1):13-24.

27. Choobineh A, Tabatabaee SH, Behzadi M. Musculoskeletal problems among workers of an Iranian sugar-producing factory. International journal of occupational safety and ergonomics. 2009 Jan 1;15(4):419-24.

28. Bugajska J, Żołnierczyk-Zreda D, Jędryka-Góral A, Gasik R, Hildt-Ciupińska K, Malińska M, Bedyńska S. Psychological factors at work and musculoskeletal disorders: a one year prospective study. Rheumatology international. 2013 Dec 1;33(12):2975-83.

29. Keyserling WM, Chaffin DB. Occupational ergonomics-methods to evaluate physical stress on the job. Annual review of public health. 1986 May;7(1):77-104.

30. Warren N. Work stress and musculoskeletal disorder etiology: The relative roles of psychosocial and physical risk factors. Work. 2001 Jan 1;17(3):221-34.

31. Leino PA. Symptoms of stress predict musculoskeletal disorders. Journal of Epidemiology \& Community Health. 1989 Sep 1;43(3):293-300.

32. Thorbjörnsson CO, Alfredsson L, Fredriksson K, Köster M, Michélsen H, Vingård E, Torgen $\mathrm{M}$, Kilbom A. Psychosocial and physical risk factors associated with low back pain: a 24 year follow up among women and men in a broad range of occupations. Occupational and environmental medicine. 1998 Feb 1;55(2):84-90.

33. Choobineh AR. Posture assessment methods in occupational ergonomics. Hamedan: Fanavaran Publication. 2004.

34. Karwowski W, Marras WS, editors. The occupational ergonomics handbook. Crc Press; 1998 Dec 18.

35. Mirmohamadi M, Seraji JN, Shahtaheri J, Lahmi M, Ghasemkhani M. Evaluation of risk factors causing musculoskeletal disorders using QEC method in a furniture producing unite. Iranian Journal of Public Health. 2004:24-7.

36. Osipow SH. Occupational stress inventory, revised edition (OSI-R). Psychological Assessment Resources, Incorporated; 1998.

37. Sharifian SA, Aminian O, Kiyani M, Barouni $\mathrm{SH}$, AMIRI F. The evaluation of the degree of occupational stress and factors influencing it in forensic physicians working in legal medicine or- ganization in Tehran-autumn of 2005.

38. Choobineh A, Daneshmandi H, Poor AF, Fard HR. Ergonomic assessment of musculoskeletal disorders risk level among workers of a petrochemical company. Iran Occupational Health. 2013 May 1;10(3).

39. Lundberg U. Stress responses in low-status jobs and their relationship to health risks: Musculoskeletal disorders. Annals of the New York Academy of Sciences. 1999 Dec;896(1):162-72.

40. Lotfizadeh M, Noor-hassim E, Habibi E. Analysis of occupational stress and the related issues among employees of Esfahan steel company (ESCO), Iran (2009). Journal of Shahrekord Uuniversity of Medical Sciences. 2011;13.

41. Givaryan H, Dindar Farkush F, Soltani SH. Factors Affecting Teacher's Job Stress and Their Management. Journal of Research in New Approach in Educational Management. 2011; (2) $3: 177-188$.

42. Spector PE, Cooper CL, Aguilar-Vafaie ME. A comparative study of perceived job stressor sources and job strain in American and Iranian managers. Applied Psychology. 2002 Jul;51(3):446-57.

43. Crawford JR, Henry J. The Depression Anxiety Stress Scales (DASS): normative data and latent structure in a large non-clinical sample. Br J Clin Psychol. 2005; 44(Pt 2): 227-39. 